\title{
The Lateral Habenula in Depression: A Mini-Review
}

\author{
Sterling Street \\ Department of Psychology, University of Georgia, Athens, Georgia, United States \\ Correspondence: sterling.street@uga.edu
}

\begin{abstract}
Although this once-obscure structure remains mysterious in many ways, the lateral habenula is receiving increasing attention as a component of the circuitry of many clinical conditions including mood disorders, anxiety disorders, pain disorders, substance use disorders, psychosis spectrum disorders, and neurodegenerative diseases. In depression research, there is growing interest in the ability of this structure to serve as a component of the brain's "anti-reward system" through its ability to inhibit the release of monoamines into regions involved in the expectation, pursuit, and receival of rewards. This mini-review covers the basics of lateral habenula structure and function, and discusses recent advances made in understanding habenular dysfunction in depression.
\end{abstract}

\section{Habenular Structure and Function}

In humans, the habenulae are two roughly symmetrical collections of neurons located on either side of the stalk of the pineal gland, near the posterior wall of the third ventricle. ${ }^{1}$ As an equivalent and less technical way to describe the same location, the habenulae are found just behind the thalamus (as part of the epithalamus), near the midline. Including both the lateral and medial division, each habenula is about the size of a pea in humans. ${ }^{2}$

Neurons in the lateral habenula receive projections from a wide range of cortical and subcortical regions. These include certain regions of the prefrontal cortex, regions of the hypothalamus (such as the paraventricular nucleus, the lateral preoptic area, the lateral hypothalamic area, and the suprachiasmatic nucleus), and various other regions such as the the ventral pallidum, the internal segment of the globus pallidus, and the bed nucleus of the stria terminalis. ${ }^{3}$

After receiving this input, neurons in the lateral habenula project glutamatereleasing axons onto GABA-releasing interneurons in the ventral tegmental area (VTA), the substantia nigra pars compacta (SNpc), the locus coeruleus (LC), the raphe nuclei, and the rostromedial tegmental nucleus. ${ }^{3,4}$ Clearly, this circuitry reveals that the lateral habenula allows a wide range of systems to influence the release of serotonin, dopamine, norepinephrine, and other signaling molecules from structures in the brain stem. 


\section{Habenular Structure and Function in Depression}

Considering the observations above, it comes as no surprise that many lines of evidence indicate that Major Depressive Disorder (hereafter depression) is associated with changes in lateral habenula structure and function. This section reviews four major lines of evidence associating depression with differences in habenular structure and function.

First, functional imaging studies have generally found increased habenular activation in depressed subjects (relative to controls) in response to aversive events such as punishment ${ }^{5}$ and reward prediction errors. ${ }^{6,7}$ For example, as measured by BOLD signal strength in fMRI, an early study found that painful shocks and monetary loss elicit a stronger habenular response in people with depression than in controls. ${ }^{8}$ Based on animal studies showing increased spiking rates in lateral habenular neurons in response to similar events, this increase in BOLD signal during such paradigms is generally interpreted as increased neuronal activity in excitatory neurons of the lateral habenula. ${ }^{8}$

Second, structural imaging studies have generally found changes in the size of the habenula between controls and people with a history of depression. However, at present there is no clear pattern regarding whether volume is generally increased or decreased; studies in different groups of patients with depression have shown both increased and decreased habenular volume relative to controls. ${ }^{9}$ For example, some MRI studies have found increased habenular volume in patients with acute, first-episode depression, ${ }^{10}$ while some postmortem studies have found decreased habenular volume. ${ }^{11}$ Further studies are needed to elucidate the precise nature of habenular volume changes in depression.

Third, a number of case reports have found that direct electrical stimulation of the lateral habenula using deep brain stimulation (DBS) is associated with a significant reduction in depression scores in some patients. ${ }^{12}$ Since it is challenging to establish causality and quantify effect size with a high degree of confidence from such reports, double-blind, sham-controlled surgical studies of lateral habenula stimulation are currently in progress. ${ }^{13}$ Further, although the mechanisms of DBS in general are not fully understood, there is evidence from animal models that stimulation by DBS can inhibit the lateral habenula by hyperpolarizing neurons in this region. ${ }^{14,15}$

Finally, the rapidly-acting antidepressant ketamine has been shown to decrease activity in the lateral habenula within timescales that seem to align with clinical response times. ${ }^{16,17}$ Although the pharmacology of ketamine is complex, ${ }^{18}$ and the mechanisms by which ketamine treats depression are not understood, it seems that blocking glutamate signaling to and from neurons in the lateral habenula may contribute to the antidepressant effects of ketamine. ${ }^{14-17}$ In other words, ketamine may treat depression partly by disinhibiting neurons in the VTA, SNpc, raphe nuclei, and other regions that are generally under negative modulation by habenular projections. 


\section{Conclusion}

This paper has described experimental and theoretical connections between the lateral habenula and Major Depressive Disorder. Lines of evidence indicating relationships between habenular activity and depressive symptoms in humans include structural and functional imaging studies, postmortem studies, pharmacological studies, and stimulation studies. A deeper understanding of this fascinating structure will greatly advance our understanding of the neurobiology of depression.

\section{References}

1 Kaye, A., \& Ross, D. A. (2017). The habenula: darkness, disappointment, and depression. Biological Psychiatry, 81(4), e27-e28.

2 Namboodiri, V. M. K., Rodriguez-Romaguera, J., \& Stuber, G. D. (2016). The habenula. Current Biology, 26(19), R873-R877.

3 Yang, Y., Wang, H., Hu, J., \& Hu, H. (2018). Lateral habenula in the pathophysiology of depression. Current Opinion in Neurobiology, 48, 90-96.

4 Chandley, M. J., \& Ordway, G. A. (2012). Noradrenergic dysfunction in depression and suicide. In The neurobiological basis of suicide. CRC Press/Taylor \& Francis.

5 Liu, W. H., Valton, V., Wang, L. Z., Zhu, Y. H., \& Roiser, J. P. (2017). Association between habenula dysfunction and motivational symptoms in unmedicated major depressive disorder. Social, Cognitive, and Affective Neuroscience, 12(9), 1520-1533.

6 Kumar, P., Goer, F., Murray, L., Dillon, D. G., Beltzer, M. L., Cohen, A. L., ... \& Pizzagalli, D. A. (2018). Impaired reward prediction error encoding and striatal-midbrain connectivity in depression. Neuropsychopharmacology, 43(7), 1581.

7 Epstein, E. L., Hurley, R. A., \& Taber, K. H. (2018). The Habenula's Role in Adaptive Behaviors: Contributions From Neuroimaging. The Journal of Neuropsychiatry and Clinical Neurosciences, 30(1), A4-4.

8 Lawson, R. P., Nord, C. L., Seymour, B., Thomas, D. L., Dayan, P., Pilling, S., \& Roiser, J. P. (2017). Disrupted habenula function in major depression. Molecular Psychiatry, 22(2), 202.

9 Boulos, L. J., Darcq, E., \& Kieffer, B. L. (2017). Translating the habenula-from rodents to humans. Biological Psychiatry, 81(4), 296-305.

10 Carceller-Sindreu, M., de Diego-Adeliño, J., Serra-Blasco, M., Vives-Gilabert, Y., Martí, A., Puigdemont, D., ... \& Portella, M. J. (2015). Volumetric MRI study of the 
habenula in first episode, recurrent and chronic major depression. European Neuropsychopharmacology, 25(11).

11 Ranft, K., Dobrowolny, H., Krell, D., Bielau, H., Bogerts, B., \& Bernstein, H. G. (2010). Evidence for structural abnormalities of the human habenular complex in affective disorders but not in schizophrenia. Psychological Medicine, 40(4), 557-567.

12 Skandalakis, G. P., Koutsarnakis, C., Kalyvas, A. V., Skandalakis, P., Johnson, E. O., $\&$ Stranjalis, G. (2018). The habenula in neurosurgery for depression: A convergence of functional neuroanatomy, psychiatry and imaging. Brain Research.

13 Sartorius, A., \& Henn, F. A. (2007). Deep brain stimulation of the lateral habenula in treatment resistant major depression. Medical Hypotheses, 69(6), 1305-1308.

14 Nuno-Perez, A., Tchenio, A., Mameli, M., \& Lecca, S. (2018). Lateral habenula gone awry in depression: bridging cellular adaptations with therapeutics. Frontiers in Neuroscience, 12.

15 Tchenio, A., Lecca, S., Valentinova, K., \& Mameli, M. (2017). Limiting habenular hyperactivity ameliorates maternal separation-driven depressive-like symptoms. Nature Communications, 8(1), 1135.

16 Yang, Y., Cui, Y., Sang, K., Dong, Y., Ni, Z., Ma, S., \& Hu, H. (2018). Ketamine blocks bursting in the lateral habenula to rapidly relieve depression. Nature, 554(7692), 317.

17 Carlson, P. J., Diazgranados, N., Nugent, A. C., Ibrahim, L., Luckenbaugh, D. A., Brutsche, N., ... \& Drevets, W. C. (2013). Neural correlates of rapid antidepressant response to ketamine in treatment-resistant unipolar depression: a preliminary positron emission tomography study. Biological Psychiatry, 73(12), 1213-1221.

18 Li, L., \& Vlisides, P. E. (2016). Ketamine: 50 years of modulating the mind. Frontiers in Human Neuroscience, 10, 612. 